\title{
Characterization and Treatment of Resistant Hypertension
}

\author{
Roberto Pisoni, MD, Mustafa I. Ahmed, MD, and David A. Calhoun, MD \\ Vascular Biology and Hypertension Program, University of Alabama at Birmingham Birmingham, \\ AL, USA
}

\begin{abstract}
Resistant hypertension is a common medical problem and carries a significantly increased risk of end organ damage and cardiovascular events as compared with more easily controlled hypertension. Resistant hypertension is most often related to isolated systolic hypertension and is characterized by aldosterone excess and increased intravascular volume. Its diagnosis requires the exclusion of pseudoresistance. The etiology of resistant hypertension is almost always multifactorial and common reversible contributing factors need to be identified and addressed. Secondary causes of hypertension such as primary aldosteronism, parenchymal and vascular kidney disease, and obstructive sleep apnea require investigation and effective treatment if present. Therapy for resistant hypertension should be based on use of rational drug class combinations at optimal doses with particular attention to adequate diuretic use. The addition of an aldosterone antagonist may further improve blood pressure control.
\end{abstract}

\section{Keywords}

resistant hypertension; blood pressure; pseudoresistance

\section{Introduction}

Resistant hypertension (RH) is defined by a failure to achieve goal blood pressure (BP) despite appropriate adherence to a three antihypertensive drug regimen [1]. It is advised but not strictly required that one of the three agents is a diuretic and all agents are prescribed at optimal doses [1]. Target $\mathrm{BP}$ is $<140 / 90 \mathrm{mmHg}$ except for those with diabetes and chronic kidney disease (CKD) where the goal is $<130 / 80 \mathrm{mmHg}$ [2]. By this definition, subjects who achieve adequate $\mathrm{BP}$ control with optimal doses of 4 or more antihypertensive medications are considered to have RH. Uncontrolled BP includes both RH and pseudoresistance where lack of BP control may be due to poor BP technique, poor adherence, white-coat effect, or inadequate treatment regimen. In this article we provide an overview of the prevalence, prognosis, patient characteristics, associated life-style factors, diagnostic evaluation, and treatment of RH.

\section{Prevalence}

The exact prevalence of RH is unknown. Data from cross-sectional and hypertension outcome studies where adherence to treatment is closely monitored, medications are provided at no charge and their titration is required per protocol, have suggested that $\mathrm{RH}$ is common, involving $20-35 \%$ of study participants. In the Antihypertensive and Lipid-Lowering Treatment to Prevent Heart Attack Trial (ALLHAT), that enrolled more than 33,000 subjects 55 years old or older with hypertension and another cardiovascular risk factor, $34 \%$ of participants had above goal $\mathrm{BP}$ on an average of 2 medications and $51 \%$ of participants required 3 or more BP

Correspondence to: Roberto Pisoni, 115 Community Health Services Building, $93319^{\text {th }}$ Street South, Birmingham, AL 35294, USA, Tel: +1205934 9281; fax +1 205934 1302. pisoni@uab.edu. 
medications [3]. In the International Verapamil-Trandolapril Study (INVEST), where more than 22,500 subjects with hypertension and known cardiovascular disease were enrolled, 29\% had BP above goal and 50\% required 3 or more BP medications [4]. The prevalence of $\mathrm{RH}$ is expected to increase due to increased life expectancy and prevalence of factors commonly associated with RH such as obesity, diabetes, and CKD.

\section{Prognosis}

Subjects with RH are at greater risk of target-organ damage such as left ventricular hypertrophy $(\mathrm{LVH})$, carotid intima-media thickening, carotid plaques, advanced retinal involvement, and albuminuria than those with more easily controlled blood pressure [5]. They have increased risk of cardiovascular events, most likely due to the long term history of poorly controlled BP and the common association of RH with diabetes, CKD, and obstructive sleep apnea (OSA) [6]. Ambulatory $\mathrm{BP}$ is a strong independent predictor of cardiovascular morbidity and mortality in RH [7]. Ambulatory systolic BP (SBP) and diastolic BP (DBP) are superior to pulse pressure (PP) as predictors, while nighttime BP is superior to daytime BP; and, surprisingly, office BP lacks prognostic value in $\mathrm{RH}$ [7]. The non-dipping pattern is also an important independent predictor of unfavorable cardiovascular outcomes in RH subjects [8]. The extent of cardiovascular risk reduction by successfully treating RH is unknown. However, the benefits of RH treatment are undoubtedly substantial as suggested by the early Veteran Administration cooperative studies where a $96 \%$ reduction in cardiovascular events was achieved over 18 months with the use of three-drug antihypertensive regimens versus placebo in subjects with DBP between 115 and $129 \mathrm{~mm} \mathrm{Hg}$ [9].

\section{Patient characteristics}

Factors associated with RH include isolated systolic hypertension (HTN), older age, aldosterone excess, increased intravascular volume, CKD, diabetes mellitus, obesity, black race, female sex, and living in the Southeastern United States [1]. Most subjects with RH have isolated systolic HTN as shown in a sub-analysis of the Framingham Heart Study where 90\% of those treated for hypertension had their DBP $<90 \mathrm{mmHg}$ while just $49 \%$ had their SBP $<140$ $\mathrm{mmHg}$ [10]. This difficulty in controlling SBP versus DBP worsens with aging [3]. Subjects with $\mathrm{RH}$ have higher plasma and urinary aldosterone levels than those with more easily controlled HTN suggesting a potentially greater role of aldosterone in causing RH than just in subjects with primary aldosteronism [11]. Higher atrial and brain natriuretic peptide levels seen in subjects with RH and without $\mathrm{CKD}$ as compared to controls suggest persistent fluid retention, which occurs despite conventional diuretic use in RH [11].

\section{Evaluation}

The evaluation of subjects with RH should aim to: distinguish true resistance from pseudoresistance; identify the factors potentially contributing to RH; identify the causes of RH with a high suspicion for secondary causes of HTN; document the degree of target organ damage; and to look for other cardiovascular risk factors. Laboratory testing of patients with RH should include serum creatinine, electrolytes, glucose, uric acid, lipids, thyroid stimulating hormone (TSH), urinalysis with evaluation for microalbuminuria, and a paired, morning plasma aldosterone concentration (PAC) and plasma renin activity (PRA) to screen for primary aldosteronism (PA), the most common secondary form of HTN. PAC/PRA (ARR) ratio >20 (with aldosterone measured in $\mathrm{ng} / \mathrm{dL}$ and PRA in $\mathrm{ng} / \mathrm{mL} / \mathrm{hr}$ ) is suggestive of PA but further evaluation is needed to confirm the diagnosis due to its poor positive prognostic value. A ARR $<20$ reliably excludes PA due to its high negative predictive value [12]. A 24-hr urine collection is recommended to confirm the diagnosis of PA and also gives useful information about the dietary sodium intake and creatinine clearance. Adrenal imaging should be done only after 
biochemical confirmation of hormonal excess. If imaging is suggestive of a tumor, it should be followed by adrenal vein sampling (AVS) to identify lateralization of aldosterone secretion consistent with an unilateral aldosterone-producing adenoma (APA) that is potentially amenable to adrenalectomy [12].

\section{Pseudoresistance}

Careful assessment of pseudoresistance avoids overtreatment and further expensive evaluations. BP should be measured after a patient has been seated quietly in a chair with his/ her back supported for five minutes, with his/her arm supported at heart level, his/her legs uncrossed, and with a properly sized cuff. The BP cuff should be placed on a bare arm to avoid any constricting action of clothing. BP should be measured in both arms and the arm with higher pressures should be used for future readings. Supine and upright BPs should be measured to detect orthostatic changes. If the cuff is too narrow or short, readings may be erroneously as high as $5-15 \mathrm{mmHg}$ in the case of SBP [13]. The presence of heavily calcified or atherosclerotic arteries that cannot be fully compressed, may lead to an overestimation of intraarterial BP in older patients. Subject should not have smoked a cigarette in the 15-30 minutes preceding the evaluation since smoking can increase SBP of up to $5-20 \mathrm{mmHg}$ [13]. It is generally recommended that coffee should be avoided although the increase in SBP following a cup of caffeinated coffee is limited to $1-2 \mathrm{mmHg}$ [13].

Poor adherence to antihypertensive therapy is an important cause of uncontrolled BP with approximately $40 \%$ of patients with newly diagnosed HTN discontinuing their antihypertensive medications during the first year of treatment [1]. The use of multiple medications and their frequent changes make adherence worse. Adherence is improved by the use of less expensive agents, with low side effect rate and once-daily dosing regimen, patient education, frequent clinic visits and by patients recording their BP at home [1]. Poor adherence is less common among patients seen by specialists versus primary care physicians.

The white-coat effect, characterized by clinic BP persistently elevated with normal or consistently lower out-of-office BP, affects 20 to $30 \%$ of the subjects with RH [1]. As in the general antihypertensive population, these subjects have less severe target organ damage and are at less cardiovascular risk compared with subjects with persistent RH during ambulatory monitoring [14]. White-coat HTN should be suspected if home BP levels reported by the patient are significantly lower than clinic BP, no target organ damage is present, and hypotension symptoms are reported despite elevated clinic BP. A 24-hour ambulatory BP measurement (ABPM) should be used to diagnose white coat effect and if that is present, the use of home $\mathrm{BP}$ values should be used to guide therapy.

Inadequate treatment regimen was the single most common cause of RH in a study evaluating 91 consecutive patients seen in a referral center [15]. This is most often due to lack of administration of more effective medications and inadequate diuretic therapy. Clinical inertia defined as a conscious reluctance by physicians to adequately treat elevated BP, contributes to this [16].

\section{Lifestyle factors}

\section{Dietary salt}

Excessive dietary sodium is common in subjects with RH and may directly increase BP and/ or blunt the antihypertensive effects of most agents including diuretics, angiotensin-converting enzyme inhibitors (ACE-Is), and angiotensin receptors blockers (ARBs). Elderly, AfricanAmericans, diabetics, and subjects with CKD appear to be at increased risk of salt sensitivity [17]. Salt restriction should be recommended to all subjects with RH and assessment of 24- 
hour urine sodium excretion should be use to assess adherence to it with a goal of $<100 \mathrm{mEq} /$ 24 hours (equivalent to 2.4 grams sodium or 6 grams sodium chloride intake) [2].

\section{Alcohol consumption}

Heavy alcohol ingestion is associated with increased BP and increased risk of BP being uncontrolled. This could be due to its physiologic effects and/or worsened adherence. In a small prospective study, cessation of heavy alcohol ingestion led to a reduction in 24-hr ambulatory SBP and DBP of 7.2 and $6.6 \mathrm{mmHg}$, respectively. Patients with RH should have their alcohol intake limited to $\leq 1$ ounce ethanol/day [1].

\section{Obesity}

Obesity is common in subjects with RH. There is a direct correlation between BMI and blood pressure with each $10 \%$ increase in body weight associated with $6.5 \mathrm{mmHg}$ increase in SBP and between BMI and number of prescribed antihypertensive medications $[18,19]$. In ALLHAT, obese subjects were achieving BP goal less than non obese subjects despite receiving more antihypertensive medications [3]. Activation of the renin-angiotensinaldosterone system (RAAS) and of the sympathetic nervous system with sodium retention, OSA, and insulin resistance play an important role in the obesity-associated HTN [1]. Weight reduction should be recommended to all overweight subjects with RH.

\section{Drug-related causes}

Multiple pharmacological agents can increase BP and contribute to BP resistance to treatment (Table 1). These include nonsteroidal anti-inflammatory drugs (NSAIDs), sympathomimetic agents such as decongestants and weight loss pills, amphetamine-like stimulants, chemotherapeutic agents, immunosuppressants such as corticosteroids and calcineurin inhibitors, oral contraceptive pills, erythropoietin stimulating agents, licorice, and over-thecounter dietary and herbal compounds [1]. There is a wide variation in the hypertensive effect of these agents with a minority of subjects being extremely sensitive to their effects. Their withdrawal should be attempted if possible. NSAIDs impair natriuresis, cause fluid retention, and may induce acute renal failure in subjects with dehydration, CKD, elderly, and diabetics where renal dysfunction can be unrecognized. NSAIDs can also blunt the effects of most antihypertensive medications. Similar effects have been shown with selective cyclooxygenase-2 (COX-2) inhibitors and appear to be mediated by their inhibition of renal prostaglandin production [1].

\section{Secondary causes}

Secondary causes of HTN account for approximately $20 \%$ of subjects referred to a HTN specialty clinic for evaluation of RH (Table 2). Primary aldosteronism (PA) is the leading cause. Older subjects are at more risk of having secondary HTN because of an increased prevalence of OSA, CKD, renal vascular disease, and PA.

\section{Primary aldosteronism}

The prevalence of PA increases according to the severity of HTN, affecting $13 \%$ of subjects with BP > 180/110 mmHg [20]. Approximately $20 \%$ of the subjects with RH were diagnosed to have PA based on a suppressed plasma renin activity and elevated 24-hour urine aldosterone excretion $(>12 \mu \mathrm{g})$ in spite of dietary sodium loading in a hypertension referral clinic at the University of Alabama at Birmingham [21]. Experimental and clinical data have shown that aldosterone excess is associated with inflammation and fibrosis, evidence of intravascular volume expansion, increased risk of target organ damage and of cardiovascular events. Hypokalemia is usually a late manifestation of the disease occurring after HTN has developed 
and should not be used to screen for PA; in our experience, $50 \%$ of subjects with RH and PA have normal serum potassium levels. PA secondary to APA responds well to resection of primary adenoma, often allowing for titration or even complete withdrawal of antihypertensives and potassium supplements while idiopathic PA is not amenable to surgical correction. In this case, long term use of aldosterone antagonists (spironolactone, eplerenone or amiloride) is the recommended treatment.

\section{Chronic kidney disease}

The presence of HTN increases linearly as the glomerular filtration rate (GFR) declines. CKD, usually resulting from diabetic nephropathy or hypertensive nephrosclerosis, is a common contributing factor to RH and also a strong predictor of failure to achieve BP goal [22]. Mechanisms involved in the pathogenesis of RH in subjects with CKD include: sodium retention, increased RAAS activity, increase sympathetic nervous system activity, and vasoconstriction secondary to hyperparathyroidism. Hypertension may also be exacerbated by the use of erythropoiesis stimulating agents in advanced CKD. RH subjects should have their GFR estimated by creatinine clearance and/or the use of Modification of Diet in Renal Disease (MDRD) Study equation, since serum creatinine is an unreliable marker of CKD, especially in the elderly [23]. They should also be assessed for the presence of albuminuria. Blockade of the RAAS slows the progression of CKD to end-stage renal disease (ESRD), reduces proteinuria and the cardiovascular risk in these patients [24].

\section{Renal artery stenosis}

Although RAS is common in hypertensive subjects undergoing cardiac catheterization with an incidence of significant lesions of $20 \%$, it is unknown what role these lesions play in causing HTN since they may be found also in normotensive subjects. Atherosclerosis accounts for $90 \%$ of the cases of renal artery stenosis (RAS). Elderly, smokers, subjects with known atherosclerotic disease are at increased risk of atherosclerotic RAS that usually presents with an exacerbation of previously controlled HTN. Flash pulmonary edema with acute renal failure after being started on an angiotensin-converting enzyme inhibitor (ACE-I), angiotensin II receptor blocker (ARB), or diuretic should raise the suspicion of bilateral RAS. Randomized clinical studies have not shown strong evidence of a benefit of surgical or endovascular revascularization versus medical management in term of improvement of renal function or BP control in these subjects, although some were able to reduce the number of antihypertensive agents after revascularization. The Cardiovascular Outcomes in Renal Atherosclerotic Lesions (CORAL) trial, a NIH-funded study to evaluate percutaneous intervention with stenting plus medical therapy versus medical therapy alone should provide important information about long-term cardiovascular and renal outcomes in atherosclerotic RAS. In the meanwhile, revascularization may be considered in cases of RH taking into account a significant BP reduction is not assured and the potential complications of the procedure such as puncture site hematoma, renal artery thrombosis or perforation, acute renal failure due to atheroembolic disease that is usually irreversible or radiocontrast agent that is usually reversible.

Fibromuscular dysplasia is a non-inflammatory, non-atherosclerotic disorder that accounts for $10 \%$ of the cases of RAS. Young women are usually affected and revascularization is successful in curing or improving HTN in most of the cases. Screening for RAS is not recommended unless the plan is to intervene if a significant stenotic lesion is found. Duplex ultrasound, magnetic resonance angiography (MRA), or computed tomography angiography (CTA) are used to screen for renovascular disease and their choice should be based upon institutional expertise and patient factors such as CKD. 


\section{Obstructive sleep apnea}

A significant direct correlation exists between severity of OSA and resistance to pharmacologic treatment of HTN $[25,26]$. OSA is very common in subjects with RH where it is more severe in men than women. Unsuspected OSA (apnea-hypopnea index $\geq 10 /$ hour) was diagnosed in $83 \%$ of 41 consecutive subjects with RH [27]. How OSA contributes to HTN is not fully understood. Increased sympathetic nervous system activity secondary to intermittent hypoxemia and increased upper airway resistance play a major contributing role as well as RAAS activation, endothelial dysfunction, and oxidative stress. A strong correlation exists between severity of OSA and aldosterone excess in subjects with RH but not in normotensive or controlled hypertensive subjects [28]. It is unclear if aldosterone excess is cause or consequence of untreated OSA. The polysomnography (PSG) is the standard criterion to diagnose and assess severity of OSA. Treatment of OSA by continuous positive airway pressure (CPAP) minimally reduced blood pressure in most published reports with the greatest benefit seen in those with more severe RH [29].

\section{Pheocromocytoma}

Pheocromocytoma is a rare cause of $\mathrm{RH}$ and accounts for 0.1 to $0.6 \%$ of subjects in a general ambulatory population. However its diagnosis and treatment are very important due to the possibility of malignancy [1]. It should be suspected in subjects with labile HTN and headache, palpitations or diaphoresis although its clinical presentation is extremely variable. Assessment of plasma free metanephrines is the preferred screening test with a sensitivity of $99 \%$ and a specificity of $89 \%$ [30]. Surgical removal is the appropriate treatment.

\section{Cushing's syndrome}

RH is common in Cushing's syndrome where severe HTN has been described in $17 \%$ of the subjects. Target organ damage is more severe in Cushing's syndrome than in primary HTN. Excessive stimulation of nonselective mineralocorticoid receptor by cortisol, OSA, diabetes, obesity, and insulin resistance contribute to HTN in these subjects [1]. The most effective BP pharmacological therapy for Cushing's syndrome is the use of an aldosterone antagonist (spironolactone or eplerenone). Surgical removal of a cortisol or adrenocorticotropic (ACTH) tumor reduces BP.

\section{Treatment of RH}

RH has almost always multifactorial in etiology. Its treatment needs to be directed at identifying and reversing lifestyle factors contributing to RH; identifying and discontinuing, if possible, medications that can contribute to RH; diagnosing and treating secondary causes of HTN; and use of effective multidrug regimens to control BP. Low salt diet, regular exercise, weight loss, moderation of alcohol intake, and quitting smoking should be routinely encouraged.

Antihypertensive agents should be prescribed at their maximum tolerated dose. Subjects whose BP remains above goal despite three or more antihypertensive medications should be referred to a hypertensive specialist. HTN specialists in a university HTN clinic, were able to achieve a BP $<140 / 90 \mathrm{mmHg}$ in $53 \%$ of subjects referred for evaluation of RH [31]. Recent data showing that bedtime dosing of antihypertensive medications was able to significantly reduce BP with greater benefit on nocturnal than diurnal HTN and to restore the non-dipping pattern in RH subjects, suggests that the use of at least one antihypertensive medication at bedtime is indicated, although this may reduce patients' adherence [32]. The efficacy of specific 3 or more drug combinations has not been evaluated in subjects with $\mathrm{RH}$ and the current recommendations are based largely on physiological principles, clinical experience, and patient characteristics (age, concomitant diseases). We often use an ACE-I (or ARB) in combination with a dihydropyridine calcium channel blocker such as amlodipine due to their better cardiovascular outcome in high risk patients and efficacy in controlling BP [33]. Since most 
of the subjects with RH have volume expansion due to excessive dietary sodium and/or sodium retention, we also prefer the use of a long acting diuretic. We prefer chlorthalidone to hydrochlorothiazide due to its greater 24-hour ambulatory BP reduction and its demonstrated outcome benefits [34,35]. A loop diuretic is preferred to a thiazide diuretic if CKD is severe (eGFR $<30-40 \mathrm{ml} / \mathrm{min} / 1.73 \mathrm{sqm}$ ) or if a potent vasodilator is used. Furosemide and bumetanide are relatively short-acting and should be prescribed at least twice daily since intermittent natriuresis with once-daily administration may lead to reactive sodium retention mediated by increases in the RAAS with subsequent inadequate BP control. Hyponatremia, hypokalemia, hyperglycemia, and hyperuricemia should be periodically monitored for in patients on diuretic therapy. If BP is still not at goal, the next step is adding a fourth agent and we typically use an aldosterone antagonist unless there is concomitant CKD (eGFR <50-60 ml/min/1.73 sqm). Spironolactone (12.5 to $50 \mathrm{mg}$ daily) decreased SBP by an additional $25 \mathrm{mmHg}$ and DBP by $12 \mathrm{mmHg}$, without racial differences, in subjects with RH already treated with at least an ACEI or ARB and a diuretic [36] and its effect was independent of serum aldosterone and renin levels. Amiloride is another potassium-sparing diuretic that is effective in reducing BP in subjects with RH [37]. Both spironolactone and amiloride were well tolerated and hyperkalemia was uncommon in patients with normal renal function. However, the risk of hyperkalemia is significantly higher in elderly subjects with CKD and/or diabetes who besides an ACE-I and/ or ARB may also regularly take a $\beta$-blocker or an NSAID. In these subjects, NSAIDS should be discontinued, spironolactone should be started at $12.5 \mathrm{mg}$ daily and serum potassium and creatinine should be closely monitored; subjects should also be educated to avoid food or supplements rich in potassium. In case the BP is still above target, we prefer combined $\alpha-\beta$ blockers such as labetalol or carvedilol to pure $\beta$-blockers due to their suggested better BP control [38]. If $\beta$ blockers are contraindicated, peripheral $\alpha$-blockers may be considered: a recent retrospective study showed that doxazosin at a mean dose of $4 \mathrm{mg}$ /day further decreased SBP by $16 \mathrm{mmHg}$ and DBP by $7 \mathrm{mmHg}$ in subjects with RH [39]. Vasodilators such as hydralazine or minoxidil and centrally acting agents such as clonidine and methyldopa may be effective but are associated with common side effects and lack of positive outcome data [2]. In particular, with minoxidil, concomitant $\beta$-blockers and loop diuretics are needed to counteract resultant reflex tachycardia and fluid retention. We do not combine an ACEI with an ARB since the combination does not significantly reduce BP or cardiovascular events as compared to individual agents and can also lead to worse renal outcome and hyperkalemia [40-42]. The ACEI/ARB combination should be limited to subjects with severe proteinuria under the care of a nephrologist. We also do not combine a renin inhibitor (aliskiren) with an ACEI or ARB since this does not generally provide substantive additional BP reduction [43]. Ongoing phase III trials are evaluating the anti-hypertensive effects of endothelin-receptor antagonists (ERAs) in RH. Cathether-based ablation of renal sympathetic nerves might be considered in the future for those subjects whose BP is persistently refractory to maximal medical treatment; this technique was safe and resulted in further decrease in SBP by $27 \mathrm{mmHg}$ and DBP by $17 \mathrm{mmHg}$ twelve months after the procedure in 45 subjects with RH [44]. Its efficacy and safety need however to be confirmed in randomized control studies as well as for interventions aimed to electrically activate the carotid baroflex system through a surgically implantable device [45].

\section{Conclusions}

$\mathrm{RH}$ is a common medical problem and its prevalence is expected to increase. RH is most often related to poorly controlled SBP. Its diagnosis requires first the use of good BP techniques to confirm the BP elevation. Other forms of pseudoresistance need to be excluded. Its etiology is almost always multifactorial and common reversible contributing factors need to be identified. The most common secondary causes of RH are PA, CKD, RAS, and OSA. Pharmacologic treatment of RH should be based on the maximum tolerated doses of multiple agents one of 
which, if possible, should be a long-acting diuretic to control volume overload. The addition of an aldosterone antagonist may further improve the BP control.

\section{References and recommended reading}

Papers of particular interest, published recently, have been highlighted as:

- Of importance

•- Of major importance

1•. Calhoun DA, Jones D, Textor S, Goff DC, Murphy TP, Toto RD, White A, Cushman WC, White W, Sica D, Ferdinand K, Giles TD, Falkner B, Carey RM. Resistant hypertension: Diagnosis, evaluation, and treatment: A scientific statement from the american heart association professional education committee of the council for high blood pressure research. Circulation 2008;117:e510e526. [PubMed: 18574054] In-depth scientific statement and review about the diagnosis, evaluation, and treatment of resistant hypertension.

2. Chobanian AV, Bakris GL, Black HR, Cushman WC, Green LA, Izzo JL Jr. Jones DW, Materson BJ, Oparil S, Wright JT Jr. Roccella EJ. Seventh report of the joint national committee on prevention, detection, evaluation, and treatment of high blood pressure. Hypertension 2003;42:1206-1252. [PubMed: 14656957]

3. Cushman WC, Ford CE, Cutler JA, Margolis KL, Davis BR, Grimm RH, Black HR, Hamilton BP, Holland J, Nwachuku C, Papademetriou V, Probstfield J, Wright JT Jr. Alderman MH, Weiss RJ, Piller L, Bettencourt J, Walsh SM. Success and predictors of blood pressure control in diverse north american settings: The antihypertensive and lipid-lowering treatment to prevent heart attack trial (allhat). J Clin Hypertens (Greenwich) 2002;4:393-404. [PubMed: 12461301]

4. Pepine CJ, Handberg EM, Cooper-DeHoff RM, Marks RG, Kowey P, Messerli FH, Mancia G, Cangiano JL, Garcia-Barreto D, Keltai M, Erdine S, Bristol HA, Kolb HR, Bakris GL, Cohen JD, Parmley WW. A calcium antagonist vs a non-calcium antagonist hypertension treatment strategy for patients with coronary artery disease. The international verapamil-trandolapril study (invest): A randomized controlled trial. Jama 2003;290:2805-2816. [PubMed: 14657064]

5. Cuspidi C, Macca G, Sampieri L, Michev I, Salerno M, Fusi V, Severgnini B, Meani S, Magrini F, Zanchetti A. High prevalence of cardiac and extracardiac target organ damage in refractory hypertension. J Hypertens 2001;19:2063-2070. [PubMed: 11677373]

6. Lewington S, Clarke R, Qizilbash N, Peto R, Collins R. Age-specific relevance of usual blood pressure to vascular mortality: A meta-analysis of individual data for one million adults in 61 prospective studies. Lancet 2002;360:1903-1913. [PubMed: 12493255]

7•. Salles GF, Cardoso CR, Muxfeldt ES. Prognostic influence of office and ambulatory blood pressures in resistant hypertension. Arch Intern Med 2008;168:2340-2346. [PubMed: 19029499] Important study regarding the prognostic value of office and ambulatory blood pressures in resistant hypertension.

8•. Muxfeldt ES, Cardoso CR, Salles GF. Prognostic value of nocturnal blood pressure reduction in resistant hypertension. Arch Intern Med 2009;169:874-880. [PubMed: 19433699] Recent study evaluating the prognostic value of nondipping pattern in resistant hypertension.

9. Effects of treatment on morbidity in hypertension. Results in patients with diastolic blood pressures averaging 115 through $129 \mathrm{~mm}$ hg. Jama 1967;202:1028-1034. [PubMed: 4862069]

10. Lloyd-Jones DM, Evans JC, Larson MG, O'Donnell CJ, Roccella EJ, Levy D. Differential control of systolic and diastolic blood pressure: Factors associated with lack of blood pressure control in the community. Hypertension 2000;36:594-599. [PubMed: 11040241]

11 •. Gaddam KK, Nishizaka MK, Pratt-Ubunama MN, Pimenta E, Aban I, Oparil S, Calhoun DA. Characterization of resistant hypertension: Association between resistant hypertension, aldosterone, and persistent intravascular volume expansion. Arch Intern Med 2008;168:1159-1164. [PubMed: 18541823] This study describes that higher aldosterone levels and greater evidence of intravascular volume expansion are present in subjects with resistant hypertension versus controls. 
12. Gonzaga CC, Calhoun DA. Resistant hypertension and hyperaldosteronism. Curr Hypertens Rep 2008;10:496-503. [PubMed: 18959838]

13. Moser M, Setaro JF. Clinical practice. Resistant or difficult-to-control hypertension. N Engl J Med 2006;355:385-392. [PubMed: 16870917]

14. Pierdomenico SD, Lapenna D, Bucci A, Di Tommaso R, Di Mascio R, Manente BM, Caldarella MP, Neri M, Cuccurullo F, Mezzetti A. Cardiovascular outcome in treated hypertensive patients with responder, masked, false resistant, and true resistant hypertension. Am J Hypertens 2005;18:14221428. [PubMed: 16280275]

15. Singer GM, Izhar M, Black HR. Goal-oriented hypertension management: Translating clinical trials to practice. Hypertension 2002;40:464-469. [PubMed: 12364348]

16. Oliveria SA, Lapuerta P, McCarthy BD, L'Italien GJ, Berlowitz DR, Asch SM. Physician-related barriers to the effective management of uncontrolled hypertension. Arch Intern Med 2002;162:413420. [PubMed: 11863473]

17. Boudville N, Ward S, Benaroia M, House AA. Increased sodium intake correlates with greater use of antihypertensive agents by subjects with chronic kidney disease. Am J Hypertens 2005;18:13001305. [PubMed: 16202852]

18. Ashley FW Jr. Kannel WB. Relation of weight change to changes in atherogenic traits: The framingham study. J Chronic Dis 1974;27:103-114. [PubMed: 4830839]

19. Modan M, Almog S, Fuchs Z, Chetrit A, Lusky A, Halkin H. Obesity, glucose intolerance, hyperinsulinemia, and response to antihypertensive drugs. Hypertension 1991;17:565-573. [PubMed: 2013483]

20. Mosso L, Carvajal C, Gonzalez A, Barraza A, Avila F, Montero J, Huete A, Gederlini A, Fardella CE. Primary aldosteronism and hypertensive disease. Hypertension 2003;42:161-165. [PubMed: 12796282]

21. Calhoun DA, Nishizaka MK, Zaman MA, Thakkar RB, Weissmann P. Hyperaldosteronism among black and white subjects with resistant hypertension. Hypertension 2002;40:892-896. [PubMed: 12468575]

22. Campese VM, Mitra N, Sandee D. Hypertension in renal parenchymal disease? Why is it so resistant to treatment? Kidney Int 2006;69:967-973. [PubMed: 16528245]

23. Levey AS, Coresh J, Balk E, Kausz AT, Levin A, Steffes MW, Hogg RJ, Perrone RD, Lau J, Eknoyan G. National kidney foundation practice guidelines for chronic kidney disease: Evaluation, classification, and stratification. Ann Intern Med 2003;139:137-147. [PubMed: 12859163]

24. Casas JP, Chua W, Loukogeorgakis S, Vallance P, Smeeth L, Hingorani AD, MacAllister RJ. Effect of inhibitors of the renin-angiotensin system and other antihypertensive drugs on renal outcomes: Systematic review and meta-analysis. Lancet 2005;366:2026-2033. [PubMed: 16338452]

25. Grote L, Hedner J, Peter JH. Sleep-related breathing disorder is an independent risk factor for uncontrolled hypertension. J Hypertens 2000;18:679-685. [PubMed: 10872551]

26. Lavie P, Hoffstein V. Sleep apnea syndrome: A possible contributing factor to resistant. Sleep 2001;24:721-725. [PubMed: 11560187]

27. Logan AG, Perlikowski SM, Mente A, Tisler A, Tkacova R, Niroumand M, Leung RS, Bradley TD. High prevalence of unrecognized sleep apnoea in drug-resistant hypertension. J Hypertens 2001;19:2271-2277. [PubMed: 11725173]

28•. Pratt-Ubunama MN, Nishizaka MK, Boedefeld RL, Cofield SS, Harding SM, Calhoun DA. Plasma aldosterone is related to severity of obstructive sleep apnea in subjects with resistant hypertension. Chest 2007;131:453-459. [PubMed: 17296647] Recent study showing a significant correlation between plasma aldosterone levels and severity of OSA in resistant hypertension but not in control subjects.

29. Robinson GV, Stradling JR, Davies RJ. Sleep . 6: Obstructive sleep apnoea/hypopnoea syndrome and hypertension. Thorax 2004;59:1089-1094. [PubMed: 15563710]

30. Lenders JW, Eisenhofer G, Mannelli M, Pacak K. Phaeochromocytoma. Lancet 2005;366:665-675. [PubMed: 16112304]

31. Garg JP, Elliott WJ, Folker A, Izhar M, Black HR. Resistant hypertension revisited: A comparison of two university-based cohorts. Am J Hypertens 2005;18:619-626. [PubMed: 15882544] 
32•. Hermida RC, Ayala DE, Fernandez JR, Calvo C. Chronotherapy improves blood pressure control and reverts the nondipper pattern in patients with resistant hypertension. Hypertension 2008;51:6976. [PubMed: 17968001] Recent study evaluating the impact of modifying the dose schedule of blood presssure treatment on the circadian pattern of blood pressure.

33••. Jamerson K, Weber MA, Bakris GL, Dahlof B, Pitt B, Shi V, Hester A, Gupte J, Gatlin M, Velazquez EJ. Benazepril plus amlodipine or hydrochlorothiazide for hypertension in high-risk patients. N Engl J Med 2008;359:2417-2428. [PubMed: 19052124] Critical study showing that benazeprilamlodipine combination was more effective than benazepril-hydrochlorothiazide combination in reducing cardiovascular events in hypertensive subjects at high risk of cardiovascular events.

34. Ernst ME, Carter BL, Goerdt CJ, Steffensmeier JJ, Phillips BB, Zimmerman MB, Bergus GR. Comparative antihypertensive effects of hydrochlorothiazide and chlorthalidone on ambulatory and office blood pressure. Hypertension 2006;47:352-358. [PubMed: 16432050]

35. Major outcomes in high-risk hypertensive patients randomized to angiotensin-converting enzyme inhibitor or calcium channel blocker vs diuretic: The antihypertensive and lipid-lowering treatment to prevent heart attack trial (allhat). Jama 2002;288:2981-2997. [PubMed: 12479763]

36. Nishizaka MK, Zaman MA, Calhoun DA. Efficacy of low-dose spironolactone in subjects with resistant hypertension. Am J Hypertens 2003;16:925-930. [PubMed: 14573330]

37. Eide IK, Torjesen PA, Drolsum A, Babovic A, Lilledahl NP. Low-renin status in therapy-resistant hypertension: A clue to efficient treatment. J Hypertens 2004;22:2217-2226. [PubMed: 15480108]

38. Townsend RR, DiPette DJ, Goodman R, Blumfield D, Cronin R, Gradman A, Katz LA, McCarthy EP, Sopko G. Combined alpha/beta-blockade versus beta 1-selective blockade in essential hypertension in black and white patients. Clin Pharmacol Ther 1990;48:665-675. [PubMed: 2249378]

39•. Rodilla E, Costa JA, Perez-Lahiguera F, Baldo E, Gonzalez C, Pascual JM. Spironolactone and doxazosin treatment in patients with resistant hypertension. Rev Esp Cardiol 2009;62:158-166. [PubMed: 19232189] Recent retrospective study showing the efficacy of spironolactone and dozaxosin in further decreasing blood pressure in subjects with resistant hypertension.

40. Stergiou GS, Makris T, Papavasiliou M, Efstathiou S, Manolis A. Comparison of antihypertensive effects of an angiotensin-converting enzyme inhibitor, a calcium antagonist and a diuretic in patients with hypertension not controlled by angiotensin receptor blocker monotherapy. J Hypertens 2005;23:883-889. [PubMed: 15775795]

41••. Yusuf S, Teo KK, Pogue J, Dyal L, Copland I, Schumacher H, Dagenais G, Sleight P, Anderson C. Telmisartan, ramipril, or both in patients at high risk for vascular events. N Engl J Med 2008;358:1547-1559. [PubMed: 18378520] This double-blind study showed that the combination of telmisartan and ramipril was associated with more adverse events without significant benefits in term of reduction of cardiovascular events versus the single agents in subjects with vascular disease or high-risk diabetes.

42. Mann JF, Schmieder RE, McQueen M, Dyal L, Schumacher H, Pogue J, Wang X, Maggioni A, Budaj A, Chaithiraphan S, Dickstein K, Keltai M, Metsarinne K, Oto A, Parkhomenko A, Piegas LS, Svendsen TL, Teo KK, Yusuf S. Renal outcomes with telmisartan, ramipril, or both, in people at high vascular risk (the ontarget study): A multicentre, randomised, double-blind, controlled trial. Lancet 2008;372:547-553. [PubMed: 18707986]

43. Oparil S, Yarows SA, Patel S, Fang H, Zhang J, Satlin A. Efficacy and safety of combined use of aliskiren and valsartan in patients with hypertension: A randomised, double-blind trial. Lancet 2007;370:221-229. [PubMed: 17658393]

44•. Krum H, Schlaich M, Whitbourn R, Sobotka PA, Sadowski J, Bartus K, Kapelak B, Walton A, Sievert H, Thambar S, Abraham WT, Esler M. Catheter-based renal sympathetic denervation for resistant hypertension: A multicentre safety and proof-of-principle cohort study. Lancet 2009;373:1275-1281. [PubMed: 19332353] Recent study evaluating the effects of cathether-based renal denervation on blood pressure in 50 patients with resistant hypertension.

45. Illig KA, Levy M, Sanchez L, Trachiotis GD, Shanley C, Irwin E, Pertile T, Kieval R, Cody R. An implantable carotid sinus stimulator for drug-resistant hypertension: Surgical technique and shortterm outcome from the multicenter phase ii rheos feasibility trial. J Vasc Surg 2006;44:1213-1218. [PubMed: 17145423] 
Table 1

Exogenous Substances that Can Contribute to Resistant Hypertension

Nonsteroidal antinflammatory agents including

Selective COX-2 inhibitors

Sympathomimetic agents (decongestants, weight loss pills, cocaine)

Stimulants (methylphenidate, amphetamine, modafinil)

Oral contraceptives

Calcineurin inhibitors

Steroids

Erythropoietin

Tricyclic antidepressant

Licorice

Alcohol

Herbal compounds (ephedra or ma huang) 
Table 2

Secondary Causes of Resistant Hypertension

Common Causes
Primary aldosteronism
Renal artery stenosis
Chronic kidney disease
Obstructive sleep apnea
Rare Causes
Pheochromocytoma
Cushing's disease
Coartation of Aorta
Intracranial tumor
Carcinoid syndrome
Hyperparathyroidism
Hypo/hyperthyroidism

